\title{
A Cross Sectional Study of Sleep Pattern among First Year Medical Students
}

\author{
Dr. Shashiraj $\mathrm{HK}^{1}$, Dr. Kavitha BS ${ }^{2 *}$, Dr. Arifuddin $\mathrm{MK}^{2}$ \\ ${ }^{1}$ Associate Professor, Department of Physiology, Sri Siddhartha Medical College, Tumkur, India \\ ${ }^{2}$ Assistant Professor, Department of Physiology, Sri Siddhartha Medical College, Tumkur, India
}

\author{
DOI: $10.36348 /$ sijap.2021.v04i03.001 \\ | Received: 03.03.2021 | Accepted: 10.03.2021 | Published: 15.03.2021 \\ *Corresponding author: Dr. Kavitha BS
}

\section{Abstract}

Background: The sleep pattern of students varies between the types of professional students (4). Medical students are a group of people in the general population who are vulnerable to poor sleep due to their long and demanding academic assignments and clinical duties including working overnight which is challenging and demanding. Sleep disturbances are known to affect the quality of work and academic performance of the students. In the last decade the rapid diffusion of gadgets especially smart phones among students and the cultural changes as led to the change in the sleep pattern of young adults. Objectives: To study the sleep pattern among first year MBBS students and to assess factors influencing sleep. Methodology: A cross sectional study was carried out among first year medical students. A self structured questionnaire was administered to all the 130 students after explaining the nature and purpose of the study. Informed consent was taken and the confidentiality was maintained during the process of this survey. The responses were analyzed using Microsoft excel and Epi Info 7. Results: $68.46 \%$ of students reported to have sleep duration of less than 6 hrs. $93.84 \%$ students of the study population agree that use of mobile phones and laptops were affecting their sleep pattern. $64.61 \%$ of the study population reported sleep latency of 30-90 min. $78.46 \%$ of study population reported waking up for 1-2 times from sleep and 55.38\% reported that it takes 10-20 min for them to return to sleep after waking up.

Keywords: Sleep pattern, medical students, duration of sleep, sleep latency, gadgets, sleep disturbances.

Copyright () 2021 The Author(s): This is an open-access article distributed under the terms of the Creative Commons Attribution 4.0 International License (CC BY-NC 4.0) which permits unrestricted use, distribution, and reproduction in any medium for non-commercial use provided the original author and source are credited.

\section{INTRODUCTION}

Sleep is defined as unconsciousness from which a person can be aroused by sensory or other stimuli. There are multiple stages of sleep from very light sleep to very deep sleep. Sleep researchers also divide sleep into two entirely different types of sleep that is rapid eye movement sleep (REM sleep) and slow-wave sleep or non-rapid eye movement sleep (NREM sleep). Sleep causes two major types of physiological effects that is on nervous system and its effect on other functional systems of the body [1].

Lack of sleep certainly does affect the functions of the central nervous system. Prolong wakefulness is often associated with progressive malfunction of the thought processes and even cause some abnormal behavioural activities. Sleep deprived people show increased sluggishness of thought that occurs towards the end of a prolonged wakeful period and in addition some people also exhibit irritability after forced wakefulness. Therefore it is assumed that sleep in multiple ways restores both normal level of brain activity and normal balance among the different functions of the central nervous system [1].

It is postulated that there is increased association of defective immune function and increased incidence of metabolic diseases in sleep deprived individuals [2]. Sleep problems are reported approximately in one third of adult population who report some form of insomnia [3].

The sleep pattern of students varies between the types of professional students [4]. Medical students are a group of people in the general population who are vulnerable to poor sleep due to their long and demanding academic assignments and clinical duties including working overnight which is challenging and demanding [5-7].

Sleep disturbances are known to affect the quality of work and academic performance of the students [4]. In the last decade the rapid diffusion of 
Shashiraj HK et al; Sch Int J Anat Physiol, Mar., 2021; 4(3): 24-26

gadgets especially smart phones among students and the cultural changes as led to the change in the sleep pattern of young adults. Hence the present study was conducted among first year medical students of Sri Siddhartha Medical College to study the sleep pattern and factors affecting sleep habits in them.

\section{MATERIALS AND METHODS}

A cross sectional study was carried out among first year medical students of the 2015-16 batch belonging to Sri Siddhartha Medical College, Tumkur, Karnataka after obtaining the approval of institutional ethical committee. A self structured questionnaire was administered to all the 130 students after explaining the nature and purpose of the study. Informed consent was taken and the confidentiality was maintained during the process of this survey. The responses were analyzed using Microsoft excel and Epi Info 7.

\section{RESULTS}

Table-1: Age and gender distribution

\begin{tabular}{|l|l|l|}
\hline & Mean & $\begin{array}{l}\text { Standard } \\
\text { deviation }\end{array}$ \\
\hline Age of the subject & 18.07 & 0.62 \\
\hline Sex & Frequency & Percentage \\
\hline Female & 60 & 46.20 \\
\hline Male & 70 & 53.80 \\
\hline
\end{tabular}

Table-2: Distribution of factors affecting sleep pattern of the study population

\begin{tabular}{|l|l|l|}
\hline $\begin{array}{l}\text { Factors affecting } \\
\text { sleep habits }\end{array}$ & Frequency & Percentage \\
\hline Caffeinated drinks intake & \multicolumn{1}{|l|}{} \\
\hline Yes & 100 & 76.92 \\
\hline No & 30 & 23.07 \\
\hline Alcohol & 2 & 1.53 \\
\hline Yes & 128 & 98.46 \\
\hline No & 3 & 2.30 \\
\hline Tobacco & 127 & 97.69 \\
\hline Yes & 57 & 43.84 \\
\hline No & 73 & 56.15 \\
\hline Regular exercise & \multicolumn{1}{|l|}{} \\
\hline Yes & 122 & 93.84 \\
\hline No & 08 & 6.15 \\
\hline Use of mobile/ laptop & 80.76 \\
\hline Yes & 105 & 19.23 \\
\hline No & 25 \\
\hline Stress & \\
\hline Yes & \\
\hline No &
\end{tabular}

Table-3: The distribution of duration of sleep in the study population

\begin{tabular}{|l|l|l|}
\hline Values & Frequency & Percentage \\
\hline Less than 6 hrs & 89 & 68.46 \\
\hline 6-8 hrs & 34 & 26.15 \\
\hline More than 8 hrs & 7 & 5.38 \\
\hline
\end{tabular}

Table-4: The distribution of sleep latency in the study population

\begin{tabular}{|l|l|l|}
\hline Values & Frequency & Percentage \\
\hline Less than 30 min & 21 & 16.15 \\
\hline 30-90 min & 84 & 64.61 \\
\hline More than 90 min & 25 & 19.23 \\
\hline
\end{tabular}

Table-5: The distribution of the sample based the number of times they wake up from sleep

\begin{tabular}{|l|l|l|}
\hline Values & Frequency & Percentage \\
\hline Not wake up & 16 & 12.30 \\
\hline One - two times & 102 & 78.46 \\
\hline 3 times or more & 12 & 9.23 \\
\hline
\end{tabular}

Table-6: The distribution of the sample based on average time usually takes to return to sleep after waking up

\begin{tabular}{|l|l|l|}
\hline Values & Frequency & Percentage \\
\hline Less than $10 \mathrm{~min}$ & 54 & 41.53 \\
\hline From $10-20 \mathrm{~min}$ & 72 & 55.38 \\
\hline More than $20 \mathrm{~min}$ & 04 & 3.07 \\
\hline
\end{tabular}

\section{DISCUSSION}

Sleep pattern is one of the phenomenons which is studied extensively worldwide because of its influence on academic routine and performance. Medical students are a group of people in the general population who are vulnerable to poor sleep due to their long and demanding academic assignments and clinical duties including working overnight which is challenging and demanding [5-7].

Table 1 which shows the age and gender distribution of our study population with the mean age of the subject being 18.07 years with a standard deviation of 0.62 . Male constitutes $53.80 \%$ (70) and females constitute $46.20 \%$ (60) of the study population.

Table 2 which depict the distribution of factors affecting sleep pattern of the study population shows that consumption of caffeinated drinks is seen in $76.92 \%$ (100) of the study population. $93.84 \%$ (122) people of the study population agree that use of mobile phones and laptops were habits which are affecting their sleep pattern. The table also shows that $80.76 \%$ (105) of people in the study population report that stress is one of the important factors which is said to have influence on the sleep pattern in them. Similar findings were reported in two other studies done by Abdul Ghani H M et al. and Giri P A et al. (c13, 14). 56.15\% (73) 
report that they don't practice any regular exercise. The table also shows that consumption of alcohol and tobacco is comparatively less in the study population and is not a factor which influences the sleep pattern in our study.

Table 3 which show the distribution of duration of sleep. It is noted that $68.46 \%$ (89) people of the study population report to having sleep duration of less than 6 hrs. $26.15 \%$ (34) report that their sleep duration is between 6-8 hrs. In a study done by Lulu A, Albhlal and et al. among medical students in Saudi Arabia showed that majority of people in their study population slept between 6-8 hrs or even less [8].

Table 4 which shows the distribution of sleep latency which is the amount of time spent in bed before falling asleep. In our study $64.61 \%$ (84) report a sleep latency of $30-90 \mathrm{~min} .19 .23 \%$ (25) report a sleep latency of more than 90 minutes. In a study done by Lulu A, Albhlal and et al. It was found that $48.4 \%$ of people had a sleep latency of 30-90 $\min$ [8].

Table 5 which shows the distribution of the sample based the number of times they wake up from sleep shows that $78.46 \%$ (102) wake up 1-2 times from sleep and $9.23 \%$ (12) wake up 3 or more times from sleep which could be because of new hostile environment.

Table 6 which shows distribution of the sample based on average time usually takes to return to sleep after waking up. In our study $55.38 \%$ (72) reported that it takes $10-20 \mathrm{~min}$ for them to return to sleep after waking up which is attributed to going back to usage of mobile phones. $41.53 \%$ (54) reports to have spent less than 10 min before going back to sleep.

\section{CONCLUSION}

In the present study we found that significantly large number of first year medical students slept less than 6 hours which could be attributed to factors like excess usage of mobile phones and excessive coffee intake along with issues related to stress. The sleep latency is significantly higher in the study population.
Our study also shows there is increase episodes of waking up among students from sleep and higher average time is also noted in going back to sleep after waking up. Based on the findings of the study it is adviced to regularly counsel the students and have student support system in place to improve the sleep quality among them and possibly prevent future development of sleep disorders and other behavioral implications which could affect their academic performance.

\section{REFERENCES}

1. Guyton, A.C., Hall, J.E. (2006). Textbook of Medical Physiology. 11th edition. Elsevier Saunders, Philadelphia.

2. Aldabal, L., Bahammam, A.S. (2011). Metabolic, endocrine, and immune consequences of sleep deprivation. Open Respir Med J, 5:31-43.

3. Mellinger, G.D., Balter, M.B., Uhlenhuth, E.H. (1958). Insomnia and its treatment. Prevalence and correlates. Arch Gen Psychiatry, 42:225-32.

4. Yang, C., Wu, C., Hsieh, H. (2003). Coping with sleep disturbances among young adults: a survey of first -year students in Taiwan. Behav Med, 29; 133-8.

5. Azad, M.C., Fraser, K., Rumana, N., Abdullah, A.F., Shahana, N. (2015). Sleep disturbances among medical students: A global perspective. $J$ Clin Sleep Med, 11:69-74.

6. Waqas, A., Khan, S., Sharif, W., Khalid, U., Ali, A. (2015). Association of academic stress with sleeping difficulties in medical students of a Pakistani medical school: A cross sectional survey. Peer J, 3:840.

7. Ibrahim, N., Dania, A. K., Lamis, E. K., Ahd, A. H., \& Asali, D. (2013). Prevalence and predictors of anxiety and depression among female medical students in King Abdulaziz University, Jeddah, Saudi Arabia. Iranian journal of public health, 42(7), 726.

8. Albhlal, L. A., Alanzi, F. G., Ghannam, K. B., Alqahtani, A., Alenazi, A. Q., \& Alanazi, M. Q. (2017). Sleep Disturbance Patterns among Medical Students, Saudi Arabia. Arch Med, 9, 3. 